\title{
Phenotypic and Molecular Convergence of 2q23.1 Deletion Syndrome with Other Neurodevelopmental Syndromes Associated with Autism Spectrum Disorder
}

\author{
Sureni V. Mullegama ${ }^{1}$, Joseph T. Alaimo ${ }^{1}$, Li Chen ${ }^{1,2}$ and Sarah H. Elsea ${ }^{1, *}$ \\ 1 Department of Molecular and Human Genetics, Baylor College of Medicine, \\ Houston, TX 77030, USA; E-Mails: mullegam@bcm.edu (S.V.M.); \\ alaimo@bcm.edu (J.T.A.); li.chen@bcm.edu (L.C.) \\ 2 Department of Cellular and Genetic Medicine, School of Basic Medical Sciences, \\ Fudan University, Shanghai 200032, China \\ * Author to whom correspondence should be addressed; E-Mail: elsea@bcm.edu; \\ Tel.: +1-713-798-5484; Fax: +1-832-825-1269.
}

Academic Editor: Merlin G. Butler

Received: 21 January 2015 / Accepted: 19 March 2015 / Published: 7 April 2015

\begin{abstract}
Roughly 20\% of autism spectrum disorders (ASD) are syndromic with a well-established genetic cause. Studying the genes involved can provide insight into the molecular and cellular mechanisms of ASD. 2q23.1 deletion syndrome (causative gene, $M B D 5)$ is a recently identified genetic neurodevelopmental disorder associated with ASD. Mutations in MBD5 have been found in ASD cohorts. In this study, we provide a phenotypic update on the prevalent features of 2 q23.1 deletion syndrome, which include severe intellectual disability, seizures, significant speech impairment, sleep disturbance, and autistic-like behavioral problems. Next, we examined the phenotypic, molecular, and network/pathway relationships between nine neurodevelopmental disorders associated with ASD: 2q23.1 deletion Rett, Angelman, Pitt-Hopkins, 2q23.1 duplication, 5q14.3 deletion, Kleefstra, Kabuki make-up, and Smith-Magenis syndromes. We show phenotypic overlaps consisting of intellectual disability, speech delay, seizures, sleep disturbance, hypotonia, and autistic-like behaviors. Molecularly, MBD5 possibly regulates the expression of $U B E 3 A, T C F 4, M E F 2 C, E H M T 1$ and RAI1. Network analysis reveals that there could be indirect protein interactions, further implicating function for these genes in common pathways. Further, we show that when MBD5 and RAII are haploinsufficient, they perturb several common pathways that are linked to neuronal and behavioral development. These
\end{abstract}


findings support further investigations into the molecular and pathway relationships among genes linked to neurodevelopmental disorders and ASD, which will hopefully lead to common points of regulation that may be targeted toward therapeutic intervention.

Keywords: $M B D 5$; ASD; networks; overlapping phenotypes; UBE3A; TCF4; $M E F 2 C$; EHMT1; RAI1; transcriptional regulation; pathways; network analysis

\section{Introduction}

Autism spectrum disorder (ASD) is a growing public health concern that affects millions of individuals worldwide [1]. ASD is a broad term encompassing a heterogeneous group of complex, highly heritable neurodevelopmental disorders in which individuals have impairments in social interaction and communication coupled with repetitive and restricted behaviors [2]. Other morbidities such as intellectual disability, epilepsy, neurological disabilities (ataxia and hypotonia), and other behavioral disorders have been associated with ASD [3]. The molecular etiology of ASD involves the interplay of many genes [4]. A contributor to the risk of ASD resides in high-impact rare variants, such as chromosomal abnormalities, copy number variation (CNV), and mutations in genes previously linked to classic monogenenic genetic disorders such as fragile X syndrome (FXS) (MIM 300624), Rett syndrome (RTT) (MIM 312750), Angelman syndrome (AS) (MIM 105830), and Smith-Magenis syndrome (SMS) (MIM 182290) [2,5,6].

With the advancement of genetic diagnostic technologies such as chromosomal microarray analysis, the identification of novel genetic subtypes of ASD and their associated genes have come to light, such as 16p11.2 deletion (MIM 611913, gene unknown), 2q23.1 deletion syndrome (MIM 156200, MBD5), Pitt-Hopkins syndrome (PTHS MIM 610954, TCF4), 5q14.3 deletion syndrome (MIM 613443, $M E F 2 C$ ), and Kleefstra syndrome (MIM 610253, EHMT1). Many of these genes are involved in neuronal functions (synaptic transmission and cell-cell interaction), chromatin modification, methylation, and transcriptional regulation [7]. The identification of these disease genes associated with ASD has led to many theories regarding the pathogenesis of ASD. One theory that has garnered support from some in the ASD community proposes that the pathogenesis is due to the disruption of neurodevelopment, which is triggered by genes with global effects on expression (chromatin modification, methylation, and transcriptional regulation) of other genes that are specifically involved in neuronal functions [7]. Further, these genes are thought to be most likely involved in common ASD-associated pathways such as cell adhesion, cadherin signaling, WNT signaling, PTEN signaling, mTOR signaling, PI3K-Akt signaling, and circadian rhythm $[4,8]$.

Corroborating studies have suggested that a disease phenotype is rarely a consequence of an abnormality in a single gene product but a reflection of dysfunction in a variety of genes and pathobiological processes that interact in complex networks and pathways [9-11]. Many neurodevelopmental genetic disorders associated with ASD share common phenotypic features despite their genetic heterogeneity, which further suggests that these genes may act together in complex interconnected pathways that, when perturbed, manifest similar phenotypes. 
2q23.1 deletion syndrome (MIM 156200), previously known as "pseudo-Angelman syndrome," was initially identified in one of the first comparative genomic hybridization (CGH) surveys of developmental disorders [12]. Patients are characterized by severe intellectual disability, seizures, significant speech impairment, and autistic-like behavioral problems [13]. 2q23.1 deletion syndrome is caused by deletion in the chromosomal region 2 q23.1 or gene specific deletions in methy-CpG-binding domain 5 (MBD5, MIM 611472). Deletions of 2q23.1 range from small deletions of $38 \mathrm{~kb}$ to $>19 \mathrm{Mb}$ [13]. While some large deletions extend in the chromosomal region $2 \mathrm{q} 22.3$, these deletions do not include the zinc finger E box-binding homeobox 2 gene (ZEB2, MIM 605802), the causative gene for Mowat-Wilson syndrome (MIM 235730). MBD5 is part of the methyl-CpG-binding domain (MBD) family, which consists of a well-known ASD-associated gene, $M E C P 2$, which is the causative gene in Rett syndrome. MBD5 has two known isoforms [14] and is thought to have a role in epigenetic modification [7,14-18]. Further, MBD5/Mbd5 has been shown to regulate expression of genes, suggesting it acts as a transcription factor $[15,17,19]$.

In this study, we conducted a comprehensive phenotypic overview of $2 \mathrm{q} 23.1$ deletion syndrome and then briefly examined the phenotypic and molecular relationships between 2q23.1 deletion syndrome and neurodevelopmental disorders associated with ASDs. Finally, we examined the pathways in common between 2q23.1 deletion syndrome and Smith-Magenis syndrome.

\section{Results and Discussion}

\subsection{2q23.1 Deletion Syndrome Clinical Review}

To further update our phenotypic knowledge of $2 \mathrm{q} 23.1$ deletion syndrome, we surveyed the most prevalent phenotypic features of all 2q23.1 deletion cases (MBD5-specific deletions and 2q23.1 deletions) using the two largest phenotypic studies on 2q23.1 deletion syndrome $[13,20]$ and case reports that were published subsequently after these studies [15,16,19,21-24]. Overall, phenotypic findings are comparable across the 74 cases reported [13,15,16,20-23,25-30], and the frequently reported neurological, neurobehavioral, and craniofacial features associated within syndrome are seen and listed in Figure 1 and Table $1[13,20]$. Neurological and behavioral features are the most prevalent findings in 2q23.1 deletion syndrome patients, while the other abnormalities, such as craniofacial abnormalities and skeletal abnormalities, are not consistently the same among individuals with this deletion (Figure 1).

Developmental delay and motor delay were present in $100 \%$ of the $2 \mathrm{q} 23.1$ deletion cases studied. Previous reports have identified seizures and severe language impairment as two primary features of $2 \mathrm{q} 23.1$ deletion syndrome, and we further show that $94.4 \%$ and $84.9 \%$ of the $2 \mathrm{q} 23.1$ deletion cases exhibit these features, respectively [13,15,16,20-23,25-30]. Infantile hypotonia and feeding difficulties were also present in greater than $85 \%$ of reported $2 \mathrm{q} 23.1$ deletion cases (Table 1). Autistic-like behaviors and behavioral problems were reported in $98.4 \%$. The most prevalent behaviors were distractibility/short attention span (100\%) and sleep disturbances (78.8\%). These studies also showed that there is a clear lack of precise documentation by clinicians regarding the exact autistic-like behaviors of individuals with 2q23.1 deletion syndrome. Nonetheless, impairments in communication, social interaction and repetitive behaviors are key behavioral criteria for ASD, which are also present in the majority of 2q23.1 deletion syndrome patients. Overall, these patients should be assessed by 
ADI-R and ADOS to identify specific ASD phenotypes present in individuals with $2 \mathrm{q} 23.1$ deletion syndrome. It is apparent that while craniofacial abnormalities are present in children with $2 \mathrm{q} 23.1$ deletion, the features are variable across the population. While mild dysmorphic craniofacial features were present at $>70 \%$ in reported cases, including broad forehead, arched/thick eyebrows, eye abnormalities, nasal abnormalities, downturned corners of the mouth, open mouth, thin upper lip, tented upper lip, and thick or everted lower lip, a consistent and thorough evaluation, measurement, and documentation of craniofacial and skeletal abnormalities is necessary for an accurate and full understanding of the commonly observed associations with 2q23.1 deletion syndrome (Figure 1).

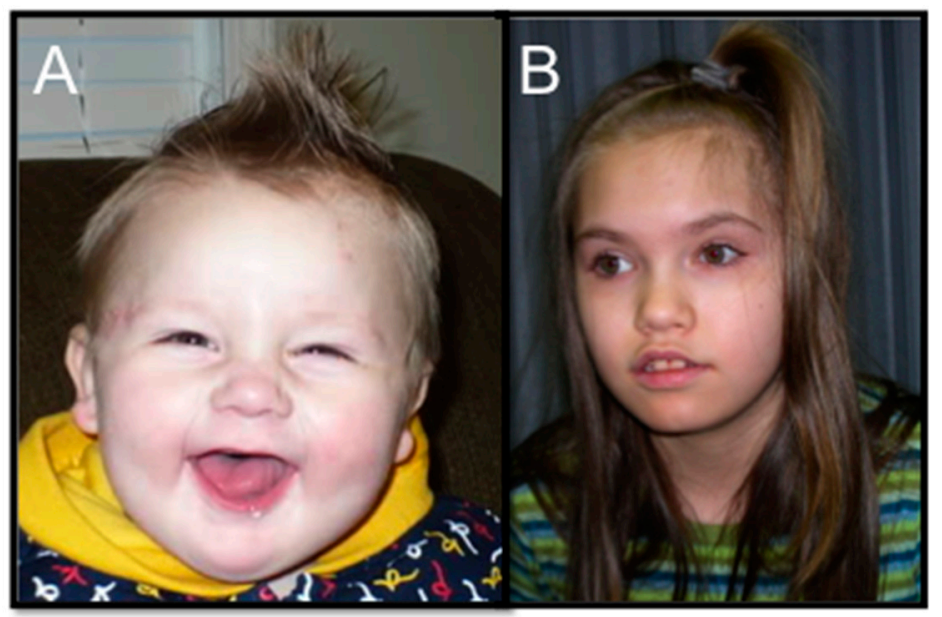

Figure 1. Craniofacial features of $2 \mathrm{q} 23.1$ deletion syndrome. Children with $2 \mathrm{q} 23.1$ deletion syndrome exhibit broad forehead, open mouth, and tented, thin upper lip. (A) 1 year old male. (B) 13 year old female. Approvals from parents were obtained to publish these photos.

Table 1. Prevalent features of 2q23.1 deletion syndrome.

\begin{tabular}{|c|c|c|}
\hline \multicolumn{3}{|c|}{ 2q23.1 Deletion ${ }^{1}$} \\
\hline Common Features & Frequency & Percentage (\%) \\
\hline \multicolumn{3}{|c|}{ Neurological } \\
\hline Developmental delay & $74 / 74$ & 100 \\
\hline Motor delay & $45 / 45$ & 100 \\
\hline Language impairment & $51 / 54$ & 94.4 \\
\hline Ataxia & $22 / 32$ & 68.7 \\
\hline Infantile hypotonia & $28 / 30$ & 93.3 \\
\hline Infantile feeding difficulties & $17 / 20$ & 85.0 \\
\hline Seizures & $45 / 53$ & 84.9 \\
\hline \multicolumn{3}{|c|}{ Behavioral } \\
\hline Autistic-like behaviors & $60 / 61$ & 98.4 \\
\hline Behavioral problems & $60 / 61$ & 98.4 \\
\hline Aggression/temper tantrums & $13 / 21$ & 62.9 \\
\hline Distractibility/short attention span & $21 / 21$ & 100 \\
\hline Hyperphagia & $8 / 16$ & 50.0 \\
\hline Self-injurious behaviors & $21 / 33$ & 63.6 \\
\hline Sleep disturbances & $41 / 52$ & 78.8 \\
\hline
\end{tabular}


Table 1. Cont.

\begin{tabular}{|c|c|c|}
\hline \multicolumn{3}{|c|}{ 2q23.1 Deletion ${ }^{1}$} \\
\hline Common Features & Frequency & Percentage $(\%)$ \\
\hline \multicolumn{3}{|c|}{ Growth/Endocrine Abnormalities } \\
\hline Postnatal growth retardation & $25 / 51$ & 49.0 \\
\hline Obesity & $6 / 17$ & 35.3 \\
\hline Short stature $(<5$ th percentile $)$ & $30 / 43$ & 69.8 \\
\hline \multicolumn{3}{|c|}{ Craniofacial Abnormalities } \\
\hline \multicolumn{3}{|l|}{ Cranium } \\
\hline Brachycephaly & $12 / 36$ & 33.3 \\
\hline Broad forehead & $21 / 30$ & 70.0 \\
\hline Microcephaly & $28 / 46$ & 60.9 \\
\hline \multicolumn{3}{|l|}{ Eyes } \\
\hline Arched/thick eyebrows & $19 / 24$ & 79.2 \\
\hline Myopia/hypermetropia/corrective lenses & $8 / 11$ & 72.7 \\
\hline Synophrys & $13 / 28$ & 46.4 \\
\hline \multicolumn{3}{|l|}{ Nose/Ear } \\
\hline Nasal abnormalities & $42 / 43$ & 97.7 \\
\hline Outer ear abnormalities & $22 / 29$ & 75.9 \\
\hline \multicolumn{3}{|l|}{ Mouth/Chin } \\
\hline Dental abnormalities & $18 / 35$ & 51.4 \\
\hline Downturned corners of the mouth & $20 / 28$ & 71.4 \\
\hline Macroglossia or protruding tongue & $8 / 33$ & 24.2 \\
\hline Micrognathia/retrognathia & $16 / 30$ & 53.3 \\
\hline Open mouth & $26 / 34$ & 76.5 \\
\hline Tented upper lip & $19 / 30$ & 63.3 \\
\hline Thin upper lip & $21 / 28$ & 75.0 \\
\hline Thick or everted lower lip & $19 / 26$ & 73.1 \\
\hline Wide mouth & $15 / 25$ & 60.0 \\
\hline \multicolumn{3}{|l|}{ Skeletal Extremity Abnormalities } \\
\hline Brachydactyly & $14 / 33$ & 42.4 \\
\hline Clinodactyly, 5th finger & $24 / 38$ & 63.2 \\
\hline Sandal gap & $12 / 32$ & 37.5 \\
\hline Short fifth digit & $16 / 37$ & 43.2 \\
\hline Small hands and feet & $25 / 37$ & 67.6 \\
\hline
\end{tabular}

${ }^{1}$ Cases came from $[13,15,20-24]$.

\subsection{Overlapping Phenotypes across 2q23.1 Deletion Syndrome and Other Autism Spectrum Disorders}

2q23.1 deletion has been aptly termed a "potent masquerader", wherein the clinical features of the disorder were initially thought to be due to other well-known genetic syndromes [20]. Since the neurodevelopmental and behavioral characteristics common to 2q23.1 deletion syndrome are nonspecific and commonly found in multiple other neurodevelopmental disorders associated with ASD [20], many 2q23.1 deletion syndrome patients were initially tested for a variety of disorders, including Rett, Angelman, and Smith-Magenis syndromes [26]. As previously mentioned, Mowat-Wilson syndrome 
and 2q23.1 deletion syndrome are on neighboring chromosome bands, patients with 2q23.1 deletion do not share key anomalies associated with Mowat-Wilson syndrome, such as Hirschsprung disease, congenital heart defects, genitourinary anomalies, and eye defects; thus, Mowat-Wilson syndrome is not in the differential diagnosis for $2 \mathrm{q} 23.1$ deletion syndrome patients [31,32]. Here, we reviewed the neurological and behavioral features of 2q23.1 deletion syndrome and eight other disorders that have been phenotypically linked to or considered in the differential diagnosis for 2q23.1 deletion syndrome [31,32]. The reported phenotypic features of 2q23.1 deletion syndrome, RTT, AS, PTHS, 2q23.1 duplication syndrome, 5q14.3 deletion syndrome, Kleefstra syndrome (KFS), Kabuki syndrome (MIM 147920), and SMS show they share neurological and behavioral co-morbidities coupled with ID, including motor impairments and gaiting abnormalities, hypotonia, language impairments, seizures, sleep disturbance, autistic-like behaviors, and other distinctive behaviors (Table 2). The similar features among many highly penetrant neurodevelopmental disorders associated with ASD allow us to hypothesize several models for ASD etiology and pathogenesis. First, the ASD-associated genes linked to known neurodevelopmental disorders govern similar cellular functions. Second, the genes play a role in specific cellular functions that converge into common molecular pathways that are associated with specific phenotypes. Third, these genes are involved in entirely different molecular pathways that converge to a common phenotype.

\subsection{MBD5 Regulates Disorder-Specific Genes}

Due to the high degree of phenotypic similarity between RTT (MECP2), AS (UBE3A), PTHS (TCF4), 2q23.1 duplication syndrome (MBD5), 5q14.3 deletion syndrome (MEF2C), KFS (EHMT1), Kabuki syndrome (KMT2D and KDM6A), and SMS (RAI1) relative to 2q23.1 deletion syndrome (Table 2), we examined the co-expression relationships of these genes to MBD5 toward assessing their involvement in the phenotype of 2q23.1 deletion syndrome. We hypothesized that these genes may be dysregulated in 2q23.1 deletion syndrome and therefore assessed expression of $M E C P 2$, UBE3A, TCF4, MEF2C, EHMT1, KMT2D, KDM6A, and RAI1 in 2q23.1 deletion syndrome patient lymphoblastoid cell lines (LCLs) that had various deletions of MBD5. As expected, mRNA levels of MBD5 were significantly down regulated in 2q23.1 deletion syndrome LCLs $(p<0.0001)$, confirming previously reported results [13] (Figure 2). Overall, we observed 5/8 (62.5\%) of the tested genes had significantly altered mRNA levels when $M B D 5$ was haploinsufficient. TCF4 and UBE3A expression levels were elevated to $\sim 1.5$-fold (TCF4, $p=0.012$; UBE3A, $p<0.0001$ ). However, MEF2C, EHMT1, and RAI1 expression levels were significantly reduced to $\sim 0.5$-fold (MEF2C, $p=0.016$; EHMT1, $p=0.0004 ; R A I 1, p<0.0001)$. MECP2, KMT2D, and KDM6A had no significant change of expression. 


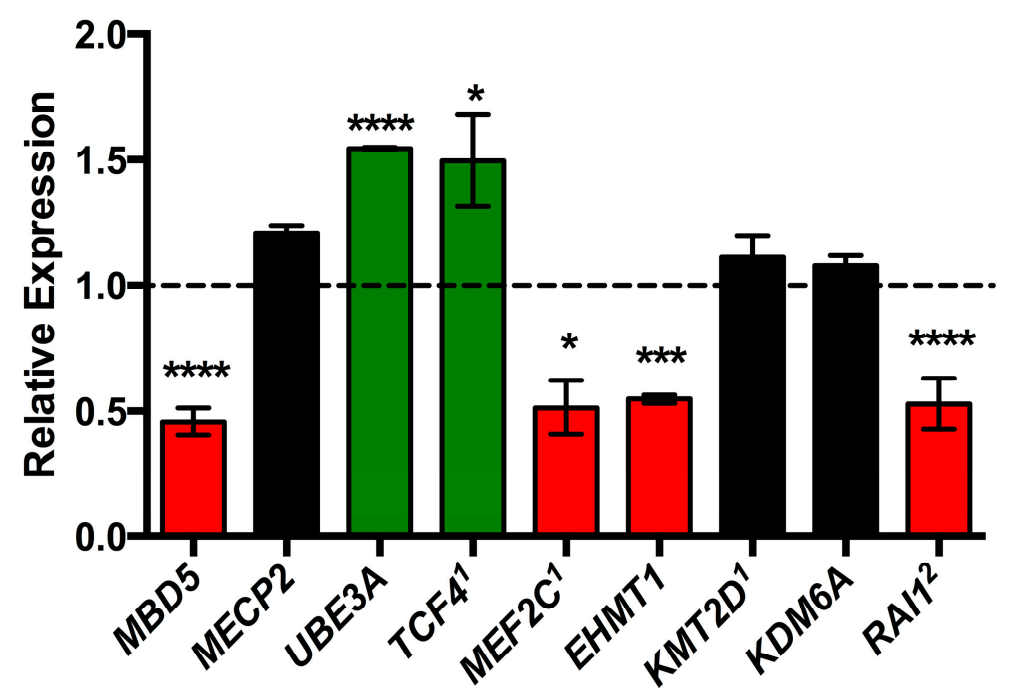

Figure 2. Dysregulation of associated genes in 2q23.1 deletion syndrome. $M B D 5, M E F 2 C$, EHTM1, and RAII were significantly down regulated (red bars) while UBE3A and TCF4 were significantly up regulated (green bars). $M E C P 2, K M T 2 D$, and KDM6A did not have altered mRNA levels (black bars). Gene expression is shown relative to control set to 1.0 (black line). Graphs represent mean $\pm \operatorname{SEM}(* p<0.05, * * * p<0.01, * * * * p<0.0001)$. $1=$ Manuscript in review, $2=[20]$.

Our co-expression data indicate that $M B D 5$ functions, either directly or indirectly, in the regulation of the expression of other ASD-associated genes (TCF4, MEF2C, EHTM1, RAI1, and UBE3A), which supports the common pathway ASD pathogenesis hypothesis. $M B D 5$ and the genes with altered expression possibly converge on common pathways that contribute to the phenotype of $2 \mathrm{q} 23.1$ deletion syndrome and genetic neurodevelopmental syndromes associated with ASD. While MECP2, KMT2D, and $K D M 6 A$, did not have altered mRNA levels, these genes may share a molecular connection to $M B D 5$ beyond co-expression, such as physical interactions. Thus, MBD5 and MECP2, KMT2D, and $K D M 6 A$ could be involved in different genetic pathways that result in a similar phenotypic output, which may indicate the presence of redundant pathways. Alternatively, MECP2, KMT2D, and KDM6A may be in the same or overlapping pathways but may function upstream of MBD5. Our phenotypic and gene expression data (Table 2 and Figure 2) implicate some form of pathway overlap or interaction when $M B D 5$ is haploinsufficient. 
Table 2. Common phenotypes between 2q23.1 deletion syndrome and Rett, Angelman, Pitt-Hopkins, 2q23.1 duplication, 5q14.3 deletion, Kleefstra, Kabuki, and Smith-Magenis syndromes.

\begin{tabular}{|c|c|c|c|c|c|c|c|c|c|}
\hline DISORDER & 2q23.1 del & RTT & AS & PTHS & $2 q 23.1$ dup & $5 q 14.3$ del & KFS & KMS & SMS \\
\hline Key References & {$[13,20]$} & {$[33,34]$} & {$[31,35]$} & {$[36,37]$} & {$[38,39]$} & [40] & [41] & {$[42,43]$} & [44] \\
\hline Causative Gene & MBD5 & $M E C P 2$ & UBE3A & TCF4 & MBD5 & $M E F 2 C$ & EHMT1 & $K M T 2 D, K D M 6 A$ & RAII \\
\hline \multicolumn{10}{|c|}{ Neurological/Behavioral Characteristics } \\
\hline Intellectual disability ${ }^{a}$ & +++ & +++ & +++ & +++ & ++ & +++ & +++ & ++ & ++ \\
\hline Speech delay ${ }^{\mathrm{b}}$ & +++ & +++ & +++ & +++ & ++ & +++ & ++ & ++ & + \\
\hline Seizures ${ }^{c}$ & +++ & +++ & +++ & ++ & ++ & ++ & ++ & + & + \\
\hline Sleep disturbance ${ }^{\mathrm{d}}$ & +++ & + & +++ & ++ & ++ & + & + & + & +++ \\
\hline Delayed walking $^{\mathrm{e}}$ & ++ & +++ & ++ & ++ & + & +++ & + & + & + \\
\hline Hypotonia & + & + & + & + & + & + & + & + & + \\
\hline Autism-like behaviors & + & + & + & + & + & + & $+^{\mathrm{f}}$ & + & + \\
\hline Feeding difficulties & + & + & + & - & + & + & + & + & + \\
\hline Stereotypic behaviors & + & + & + & + & + & + & - & + & + \\
\hline Ataxia & + & + & + & + & + & + & - & + & - \\
\hline $\begin{array}{l}\text { Happy disposition (frequent } \\
\text { or inappropriate laughing) }\end{array}$ & + & + & + & + & + & NR & - & + & - \\
\hline $\begin{array}{c}\text { Hyperactivity/ } \\
\text { short attention span }\end{array}$ & + & - & + & + & + & - & - & + & + \\
\hline Self-injurious behavior & + & - & - & - & - & - & + & - & + \\
\hline Aggressive behavior & - & - & - & + & - & - & + & - & + \\
\hline
\end{tabular}

2q23.1 del = 2q23.1 deletion syndrome, RTT = Rett syndrome, AS = Angelman syndrome, PTHS = Pitt-Hopkins syndrome, 2q23.1 dup = 2q23.1 duplication syndrome, $5 \mathrm{q} 14.3 \mathrm{del}=5 \mathrm{q} 14.3$ deletion syndrome, $\mathrm{KFS}=$ Kleefstra syndrome, KMS $=$ Kabuki make-up syndrome, and Smith-Magenis syndrome, ${ }^{\mathrm{a}}+=$ mild; $++=$ moderate; $+++=$ severe, ${ }^{\mathrm{b}}+=$ moderate; $++=$ severe; $+++=$ absent,${ }^{\mathrm{c}}+=0 \%-40 \% ;++=41 \%-70 \% ;+++71 \%-100 \%,{ }^{\mathrm{d}}+=0 \%-40 \% ;++40 \%-70 \% ;+++71 \%-100 \%,{ }^{\mathrm{e}}+=1-3 \mathrm{y}$; $++=4-6 \mathrm{y} ;+++=>6$ y or limited mobility, ${ }^{\mathrm{f}}$ only in childhood, $\mathrm{NR}=$ not reported. 


\subsection{MBD5 Network}

Recent studies suggest that genes involved in common endpoints (e.g., phenotypes) show an increased tendency to have protein-protein interactions, similar expression patterns in specific tissues, and exhibit synchronized expression as a group $[45,46]$. In this study, we observed co-expression relationships between MBD5 and ASD-associated genes (Figure 2). To comprehensively understand MBD5-dependent genetic pathways relative to these genes, we utilized bioinformatics resources and the network tool, Cognoscente (http://vanburenlab.tamhsc.edu/), to uncover related protein interactions (Figure 3). While data do not exist in the literature to support the key genes (in red boxes) directly interacting with each other, we did observe that some hubs (central network connections) all intervened with one another (Figure 3). This finding could suggest there are intermediary genes that molecularly connect the genes directly involved in known disorders. Interestingly, from this network, several other genes linked to ASD were identified, including CDKL5, HDAC4, EP300, FMR1, $S M A R C A 4$, and $A T R X$, each previously linked to a neurodevelopmental disorder with overlapping or similar phenotypes, as listed in Table 2 (Figure 2). In Mullegama et al. (2014), we observed overexpression of FMR1 in 2q23.1 deletion syndrome cell lines [19]. Additional expression studies of these ASD-associated genes in 2 q23.1 deletion syndrome would prove interesting. A limitation using such tools to explore molecular networks, as we demonstrated in Figure 2, is that all programs that generate networks, like Cognoscente, rely on the published literature [47]. This fact revealed that there is clearly a lack of research and understanding of the interactions among the genome and proteome in ASD. Thus, undoubtedly, concerted efforts need to be made to study molecular interactions between these genes collectively to further elucidate the genetic etiology of these and other monogenic neurodevelopmental disorders associated with ASDs. Futures studies such as RNA-seq, methylation sequencing, whole-genome miRNA analysis, and chromatin immunoprecipitation sequencing (ChIP-seq) studies of these disorder-causing genes will allow us to further elucidate the levels of molecular convergence between these disorders.

\subsection{Molecular Relationships between MBD5 and RAII}

We propose that functional relationships between genes serve as a strong indicator for involvement in key pathways responsible for phenotypic outcomes. An example of this concept is the relationship between MBD5 and RAI1. We have shown that 2q23.1 deletion syndrome and Smith-Magenis syndrome have overlapping phenotypes (see Table 2). From the data we presented here, it appears that $R A I 1$ could be regulated directly or indirectly by MBD5. Our previously published work also suggested that $R A I 1$ is dysregulated when MBD5 is knocked down through siRNA technology in neuroblastoma SH-SY5Y cell lines [19]. Therefore, we wanted to further examine the functional pathways common to $M B D 5$ and RAII. 


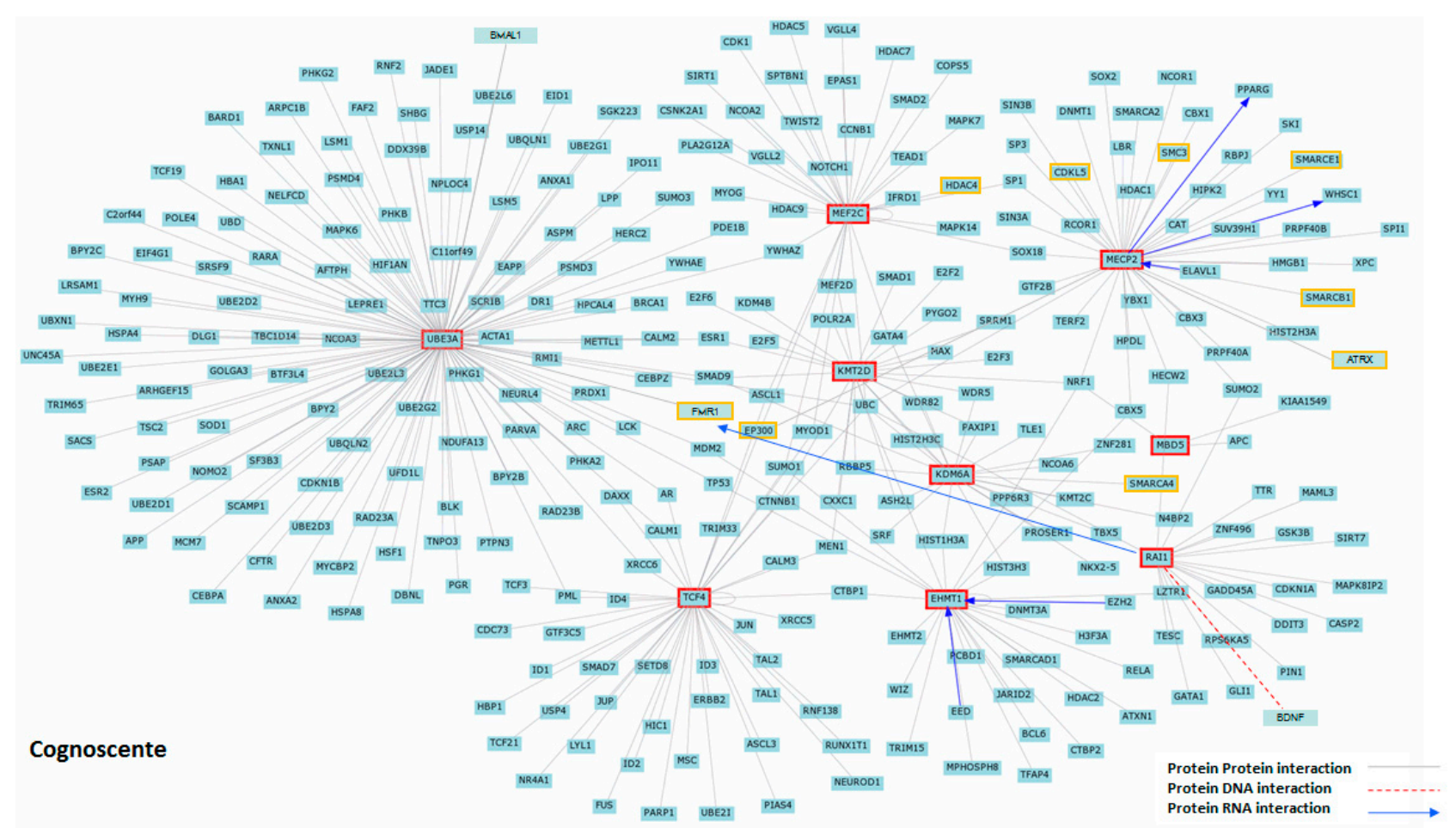

Figure 3. 2q23.1 deletion syndrome gene interaction network. Cognoscente was used to generate direct protein-protein (grey line), protein-DNA (red dash line), and protein-RNA (blue arrow line) interactions of genes previously implicated in ASD: $M B D 5, M E C P 2$, UBE3A, TCF4, MEF2C, EHMT1, KMT2D, KDM6A and RAI1 (red box). The human orthologs of genes of many of the protein products that came up in this network are involved in neuronal functions, chromatin modification, methylation and transcriptional regulation. In addition, other genes associated with ASD also were identified (yellow box).

From previously published microarray data [19] where MBD5 and RAII were knocked down through siRNA technology individually in neuroblastoma cell lines, we conducted Ingenuity Pathway Analysis (IPA) on these microarray data to identify the common pathways that are perturbed when $M B D 5$ and $R A I 1$ are haploinsufficient. We propose that these pathways could be key contributors to the overlapping phenotypes we see in both syndromes. IPA was used to determine which biological pathways and function involving genes were differentially expressed in MBD5 and RAI1 knockdown SH-SY5Y cell lines. A $p$-value of less than 0.05 for each pathway was determined using Fisher's exact tests to determine the likelihood of those genes assigned by chance. IPA shows that MBD5 and RAI1 are implicated in many neurological, cell growth and developmental pathways $(p<0.05)$ (Tables 3 and 4). The top common pathways reveal many interesting and important pathways altered due to MBD5 and RAI1 haploinsufficiency. Previously mentioned pathways that are associated with autism and sleep, circadian rhythm signaling, and mTOR signaling pathways are present (Tables 3 and 4) [19]. The CDK5 signaling pathway is crucial to neuronal activity, neuronal migration during development and neurite growth [48]. In previous mouse studies of Mbd5, reduced neurite outgrowth was observed [49]. Further, the Xenopus laevis rail morphants exhibit aberrant neural crest migration [50]. Thus, the CDK5 signaling pathway could be contributing to the features seen in the above studies. The apoptosis signaling pathway (see Table 4) is involved in the determination of the size and shape of the brain and 
it regulates the wiring of developing neuronal networks [51]. It is thought that dysregulation of this pathway can lead to neuroanatomic abnormalities and developmental disabilities [51]. There are growing associations between neural cell death and autism [51]. Thus, the involvement of the apoptosis signaling pathway in 2q23.1 deletion syndrome and Smith-Magenis syndrome should be further investigated. Overall, extensive molecular studies on how these shared pathways contribute to the phenotypes present in 2q23.1 deletion syndrome and Smith-Magenis syndrome is necessary. In addition, these results provide further evidence that monogenic neurodevelopmental disorders associated with ASD share common pathways and are crucial to study in regards to phenotypic development.

Table 3. Common neurological pathways in both MBD5 and RAI1 knockdown SH-SY5Y cells.

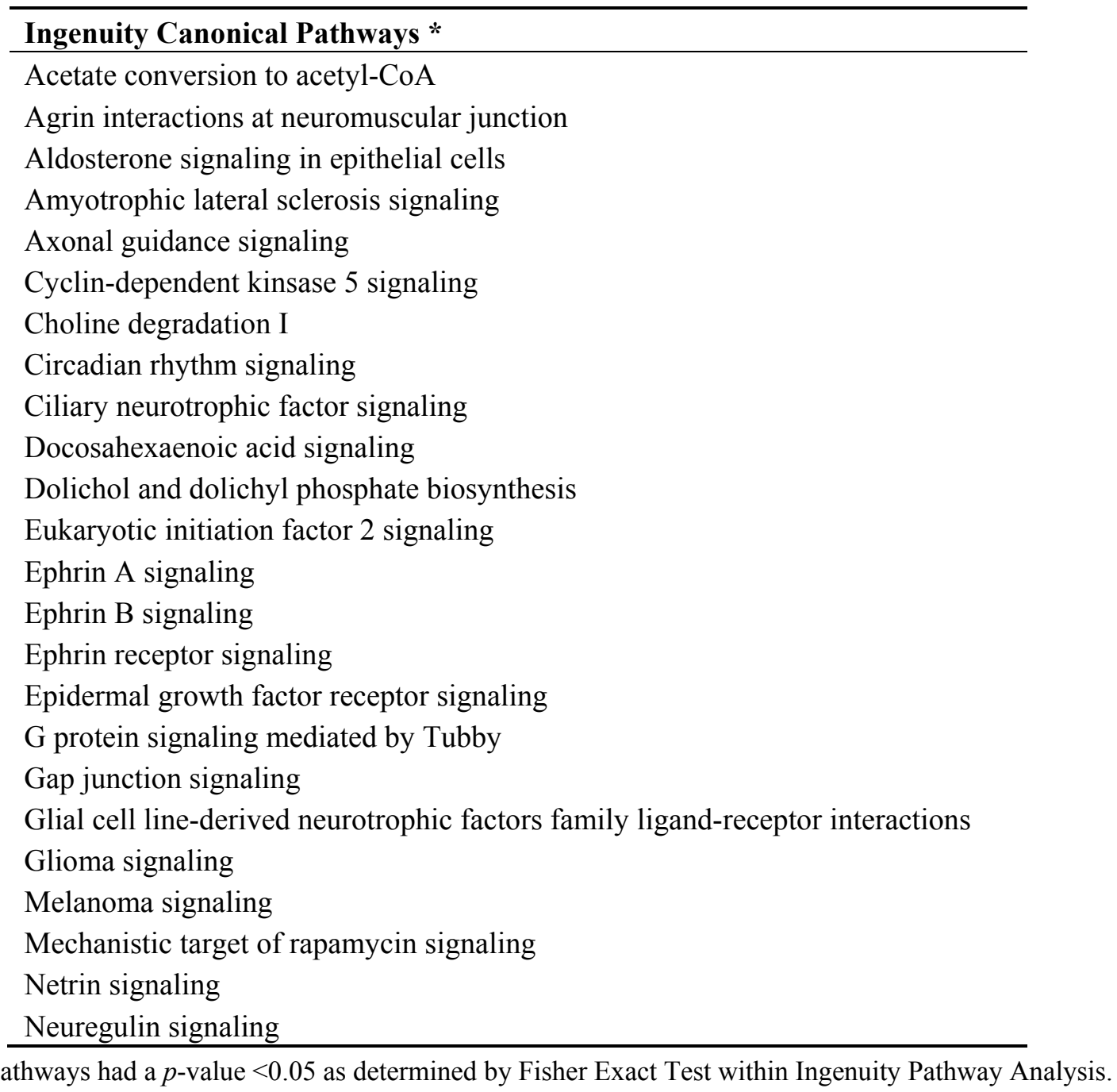


Table 4. Common cell growth and developmental pathways in both MBD5 and RAII knockdown SH-SY5Y cells.

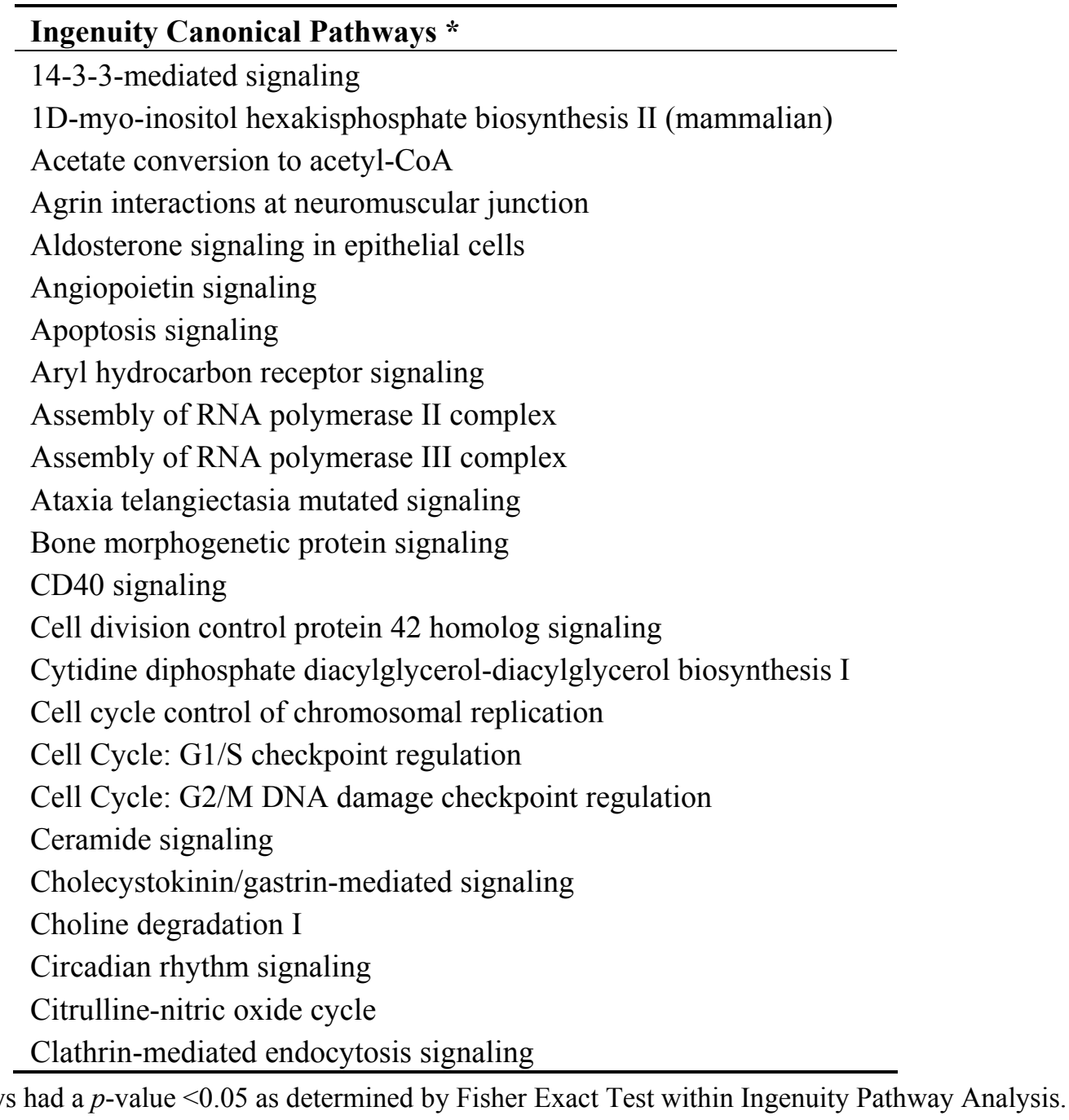

\section{Experimental Section}

\subsection{Patients and Cell Culture Studies}

All samples and information were collected after informed consent was obtained and in accordance with Institutional Review Board (IRB) approved protocols from Baylor College of Medicine. Lymphoblastoid cell lines (LCLs) (Epstein-Barr virus-transformed human lymphocytes) were cultured as previously described [25]. LCLs utilized in this study included: 2q23.1 deletion syndrome (SMS367 [25], SMS185 [25], SMS361 [13], SMS373 [13], SMS375 [13], and SMS368 [13] and five normal control lines).

\subsection{Expression Analyses}

RNA was isolated from cultured patient and control lymphoblastoid cell lines via TRIzol (Invitrogen, Carlsbad, CA, USA) according to standard protocols. All cell lines were cultured for the same period of time to the same cell density. RNA was quantified using the NanoDrop ${ }^{\circledR}$ ND-100 Spectrophotometer (NanoDrop Technologies, Inc., Wilmington, DE, USA). First-strand cDNA 
synthesis was carried out using qSCRIPT cDNA SuperMix (Quanta Biosciences, Inc., Gaithersburg, MD, USA) (with $1 \mu \mathrm{g}$ of RNA) according to the manufacturer's protocol. For quantitative real-time PCR, predesigned Taqman MGB probes from Assays-on-Demand Gene Expression Products (ABI) (Life Technologies Inc., Carlsbad, CA, USA) were used for all genes. A minimum of three unrelated patient samples were run in triplicate in $10 \mu \mathrm{L}$ reaction volumes. PCR conditions were the default settings of the ABI Prism 7900 HT Sequence Detection System (Life Technologies Inc., Carlsbad, CA, USA). The cycle threshold $\left(C_{\mathrm{t}}\right)$ was determined during the geometric phase of the PCR amplification plots as recommended by the manufacturer. Relative differences in transcript levels were quantified with the $\triangle \triangle C$ t method with GAPDH (MIM138400) (Hs99999905_m1) mRNA as an endogenous control. All expression values were calculated relative to control levels set at 1.0.

\subsection{Network Analysis}

Cognoscente (http://vanburenlab.tamhsc.edu/) was used to identify the biomolecular interactions that have been documented in the literature of the genes listed in Table 2. Cognoscente is a highly curated freely available database that identifies interacting components of protein networks and the primary literature to support such interactions [47].

\subsection{Ingenuity Pathway Analysis (IPA)}

IPA was used to identify biological functions, gene networks and pathways, and likely upstream regulators that were significantly more altered in knockdown cells than in controls. Significant interactions were determined using the Ingenuity Pathway Knowledge Base and a Fisher's exact test to calculate a $p$-value determining the probability that each function network or pathway assigned to that data set is due to chance alone. Statistical significance was determined at a cutoff of $p \leq 0.05$.

\subsection{Statistical Analyses}

Statistical analysis for gene expression data was performed with Prism 4 version 4.0b (GraphPad Software, Inc., San Diego, CA, USA). Statistical significance was determined at a cutoff of $p \leq 0.05$.

\section{Conclusions}

In conclusion, we show that $2 \mathrm{q} 23.1$ deletion syndrome shares common neurological and behavioral phenotypes with other monogenic neurodevelopmental disorders associated with autism spectrum disorders. Furthermore, haploinsufficiency of MBD5 impacts expression of several ASD-implicated genes, including UBE3A, TCF4 MEF2C, EHMT1, and RAII; supporting MBD5 as a transcriptional regulator. Further, data suggest that these genes associated with ASD, MBD5, MECP2, UBE3A, TCF4 $M E F 2 C, E H M T 1, K M T 2 D$ and KDM6A, and RAI1 may be part of a gene network that converges into common or overlapping pathways that results in similar phenotypes when perturbed. As an example, we show that when $M B D 5$ and $R A I 1$ are both haploinsufficient they share common perturbed pathways that likely contribute to the overlapping phenotypes exhibited by 2 q23.1 deletion syndrome and Smith-Magenis syndrome patients. Overall, future studies identifying and dissecting the genetic and downstream molecular pathways in monogenic neurodevelopmental disorders associated with ASD 
may reveal common points of regulation and promote gene candidates for targeted therapeutic and pharmacological intervention.

\section{Acknowledgments}

We are deeply indebted to the study participants and their families, as well as support from the Fondation Jérôme Lejeune. This work was supported in part by the resources from Baylor College of Medicine, the Jan and Dan Duncan Neurological Research Institute, and the Texas Children's Hospital. Li Chen was funded by the National Science Foundation-China (NSFC) grant 31200937 and Shanghai Health and Family Planning Commission grant 20144Y0106.

\section{Author Contributions}

Sureni V. Mullegama conceived the study, conducted the experiments, analyzed the data and wrote the manuscript. Joseph T. Alaimo and Li Chen helped create figures and critically edited the manuscript. Sarah H. Elsea is the principal investigator and critically edited the manuscript.

\section{Conflicts of Interest}

The authors declare no conflicts of interest.

\section{References}

1. Spooren, W.; Lindemann, L.; Ghosh, A.; Santarelli, L. Synapse dysfunction in autism: A molecular medicine approach to drug discovery in neurodevelopmental disorders. Trends Pharmacol. Sci. 2012, 33, 669-684.

2. Ceroni, F.; Sagar, A.; Simpson, N.H.; Gawthrope, A.J.; Newbury, D.F.; Pinto, D.; Francis, S.M.; Tessman, D.C.; Cook, E.H.; Monaco, A.P.; et al. A deletion involving CD38 and BST1 results in a fusion transcript in a patient with autism and asthma. Autism Res. 2014, 7, 254-263.

3. Pendergrass, S.; Girirajan, S.; Selleck, S. Uncovering the etiology of autism spectrum disorders: Genomics, bioinformatics, environment, data collection and exploration, and future possibilities. Pac. Symp. Biocomput. 2014, 422-426.

4. De Rubeis, S.; He, X.; Goldberg, A.P.; Poultney, C.S.; Samocha, K.; Cicek, A.E.; Kou, Y.; Liu, L.; Fromer, M.; Walker, S.; et al. Synaptic, transcriptional and chromatin genes disrupted in autism. Nature 2014, 515, 209-215.

5. Marshall, C.R.; Noor, A.; Vincent, J.B.; Lionel, A.C.; Feuk, L.; Skaug, J.; Shago, M.; Moessner, R.; Pinto, D.; Ren, Y.; et al. Structural variation of chromosomes in autism spectrum disorder. Am. J. Hum. Genet. 2008, 82, 477-488.

6. Betancur, C. Etiological heterogeneity in autism spectrum disorders: More than 100 genetic and genomic disorders and still counting. Brain Res. 2011, 1380, 42-77.

7. Blumenthal, I.; Ragavendran, A.; Erdin, S.; Klei, L.; Sugathan, A.; Guide, J.R.; Manavalan, P.; Zhou, J.Q.; Wheeler, V.C.; Levin, J.Z.; et al. Transcriptional consequences of 16p11.2 deletion and duplication in mouse cortex and multiplex autism families. Am. J. Hum. Genet. 2014, 94, 870-883. 
8. Pasciuto, E.; Bagni, C. Snapshot: Fmrp interacting proteins. Cell 2014, 159, 218-218.e1.

9. Van Bokhoven, H. Genetic and epigenetic networks in intellectual disabilities. Annu. Rev. Genet. 2011, 45, 81-104.

10. Urdinguio, R.G.; Sanchez-Mut, J.V.; Esteller, M. Epigenetic mechanisms in neurological diseases: Genes, syndromes, and therapies. Lancet Neurol. 2009, 8, 1056-1072.

11. Oti, M.; Huynen, M.A.; Brunner, H.G. Phenome connections. Trends Genet. 2008, 24, 103-106.

12. Vissers, L.E.; de Vries, B.B.; Osoegawa, K.; Janssen, I.M.; Feuth, T.; Choy, C.O.; Straatman, H.; van der Vliet, W.; Huys, E.H.; van Rijk, A.; et al. Array-based comparative genomic hybridization for the genomewide detection of submicroscopic chromosomal abnormalities. Am. J. Hum. Genet. 2003, 73, 1261-1270.

13. Talkowski, M.E.; Mullegama, S.V.; Rosenfeld, J.A.; van Bon, B.W.; Shen, Y.; Repnikova, E.A.; Gastier-Foster, J.; Thrush, D.L.; Kathiresan, S.; Ruderfer, D.M.; et al. Assessment of 2q23.1 microdeletion syndrome implicates MBD5 as a single causal locus of intellectual disability, epilepsy, and autism spectrum disorder. Am. J. Hum. Genet. 2011, 89, 551-563.

14. Laget, S.; Joulie, M.; le Masson, F.; Sasai, N.; Christians, E.; Pradhan, S.; Roberts, R.J.; Defossez, P.A. The human proteins MBD5 and MBD6 associate with heterochromatin but they do not bind methylated DNA. PLoS ONE 2010, 5, e11982.

15. Bonnet, C.; Ali Khan, A.; Bresso, E.; Vigouroux, C.; Beri, M.; Lejczak, S.; Deemer, B.; Andrieux, J.; Philippe, C.; Moncla, A.; et al. Extended spectrum of mbd5 mutations in neurodevelopmental disorders. Eur. J. Hum. Genet. 2013, 21, 1457-1461.

16. Cukier, H.N.; Lee, J.M.; Ma, D.; Young, J.I.; Mayo, V.; Butler, B.L.; Ramsook, S.S.; Rantus, J.A.; Abrams, A.J.; Whitehead, P.L.; et al. The expanding role of mbd genes in autism: Identification of a mecp2 duplication and novel alterations in mbd5, mbd6, and setdb1. Autism Res. 2012, 5, 385-397.

17. Du, Y.; Liu, B.; Guo, F.; Xu, G.; Ding, Y.; Liu, Y.; Sun, X.; Xu, G. The essential role of MBD5 in the regulation of somatic growth and glucose homeostasis in mice. PLoS ONE 2012, 7, e47358.

18. Ladha, S. Getting to the bottom of autism spectrum and related disorders: MBD5 as a key contributor. Clin. Genet. 2012, 81, 363-364.

19. Mullegama, S.V.; Pugliesi, L.; Burns, B.; Shah, Z.; Tahir, R.; Gu, Y.; Nelson, D.L.; Elsea, S.H. MBD5 haploinsufficiency is associated with slee disturbance and disrupts circadian pathways common to smith-magenis and fragile $\mathrm{X}$ syndromes. Eur J. Hum. Genet. 2014, doi:10.1038/ejhg.2014.200.

20. Hodge, J.C.; Mitchell, E.; Pillalamarri, V.; Toler, T.L.; Bartel, F.; Kearney, H.M.; Zou, Y.S.; Tan, W.H.; Hanscom, C.; Kirmani, S.; et al. Disruption of mbd5 contributes to a spectrum of psychopathology and neurodevelopmental abnormalities. Mol. Psychiatry 2014, 19, 368-379.

21. Du, X.; An, Y.; Yu, L.; Liu, R.; Qin, Y.; Guo, X.; Sun, D.; Zhou, S.; Wu, B.; Jiang, Y.H.; et al. A genomic copy number variant analysis implicates the mbd5 and hnrnpu genes in chinese children with infantile spasms and expands the clinical spectrum of 2q23.1 deletion. BMC Med. Genet. 2014, 15, 62 .

22. Lund, C.; Brodtkorb, E.; Rosby, O.; Rodningen, O.K.; Selmer, K.K. Copy number variants in adult patients with lennox-gastaut syndrome features. Epilepsy Res. 2013, 105, 110-117. 
23. Shichiji, M.; Ito, Y.; Shimojima, K.; Nakamu, H.; Oguni, H.; Osawa, M.; Yamamoto, T. A cryptic microdeletion including MBD5 occurring within the breakpoint of a reciprocal translocation between chromosomes 2 and 5 in a patient with developmental delay and obesity. Am. J. Med. Genet. A 2013, 161, 850-855.

24. Girirajan, S.; Dennis, M.Y.; Baker, C.; Malig, M.; Coe, B.P.; Campbell, C.D.; Mark, K.; Vu, T.H.; Alkan, C.; Cheng, Z.; et al. Refinement and discovery of new hotspots of copy-number variation associated with autism spectrum disorder. Am. J. Hum. Genet. 2013, 92, 221-237.

25. Williams, S.R.; Mullegama, S.V.; Rosenfeld, J.A.; Dagli, A.I.; Hatchwell, E.; Allen, W.P.; Williams, C.A.; Elsea, S.H. Haploinsufficiency of mbd5 associated with a syndrome involving microcephaly, intellectual disabilities, severe speech impairment, and seizures. Eur. J. Hum. Genet. 2010, 18, 436-441.

26. Van Bon, B.W.; Koolen, D.A.; Brueton, L.; McMullan, D.; Lichtenbelt, K.D.; Ades, L.C.; Peters, G.; Gibson, K.; Moloney, S.; Novara, F.; et al. The 2q23.1 microdeletion syndrome: Clinical and behavioural phenotype. Eur J. Hum. Genet. 2010, 18, 163-170.

27. Noh, G.J.; Graham, J.M., Jr. 2q23.1 microdeletion of the MBD5 gene in a female with seizures, developmental delay and distinct dysmorphic features. Eur. J. Med. Genet. 2012, 55, 59-62.

28. Motobayashi, M.; Nishimura-Tadaki, A.; Inaba, Y.; Kosho, T.; Miyatake, S.; Niimi, T.; Nishimura, T.; Wakui, K.; Fukushima, Y.; Matsumoto, N.; et al. Neurodevelopmental features in 2q23.1 microdeletion syndrome: Report of a new patient with intractable seizures and review of literature. Am. J. Med. Genet. A 2012, 158, 861-868.

29. Chung, B.H.; Stavropoulos, J.; Marshall, C.R.; Weksberg, R.; Scherer, S.W.; Yoon, G. 2q23 de novo microdeletion involving the mbd5 gene in a patient with developmental delay, postnatal microcephaly and distinct facial features. Am. J. Med. Genet. A 2011, 155, 424-429.

30. Wagenstaller, J.; Spranger, S.; Lorenz-Depiereux, B.; Kazmierczak, B.; Nathrath, M.; Wahl, D.; Heye, B.; Glaser, D.; Liebscher, V.; Meitinger, T.; et al. Copy-number variations measured by single-nucleotide-polymorphism oligonucleotide arrays in patients with mental retardation. Am. J. Hum. Genet. 2007, 81, 768-779.

31. Tan, W.H.; Bird, L.M.; Thibert, R.L.; Williams, C.A. If not angelman, what is it? A review of angelman-like syndromes. Am. J. Med. Genet. A 2014, 164, 975-992.

32. Kleefstra, T.; Kramer, J.M.; Neveling, K.; Willemsen, M.H.; Koemans, T.S.; Vissers, L.E.; Wissink-Lindhout, W.; Fenckova, M.; van den Akker, W.M.; Kasri, N.N.; et al. Disruption of an ehmt1-associated chromatin-modification module causes intellectual disability. Am. J. Hum. Genet. 2012, 91, 73-82.

33. Smeets, E.E.; Pelc, K.; Dan, B. Rett syndrome. Mol. Syndromol. 2012, 2, 113-127.

34. Neul, J.L. The relationship of Rett syndrome and MECP2 disorders to autism. Dialogues Clin. Neurosci. 2012, 14, 253-262.

35. Kyllerman, M. Angelman syndrome. Handb. Clin. Neurol. 2013, 111, 287-290.

36. Whalen, S.; Heron, D.; Gaillon, T.; Moldovan, O.; Rossi, M.; Devillard, F.; Giuliano, F.; Soares, G.; Mathieu-Dramard, M.; Afenjar, A.; et al. Novel comprehensive diagnostic strategy in Pitt-hopkins syndrome: Clinical score and further delineation of the TCF4 mutational spectrum. Hum. Mutat. 2012, 33, 64-72. 
37. Marangi, G.; Ricciardi, S.; Orteschi, D.; Tenconi, R.; Monica, M.D.; Scarano, G.; Battaglia, D.; Lettori, D.; Vasco, G.; Zollino, M. Proposal of a clinical score for the molecular test for Pitt-hopkins syndrome. Am. J. Med. Genet. A 2012, 158, 1604-1611.

38. Chung, B.H.; Mullegama, S.; Marshall, C.R.; Lionel, A.C.; Weksberg, R.; Dupuis, L.; Brick, L.; Li, C.; Scherer, S.W.; Aradhya, S.; et al. Severe intellectual disability and autistic features associated with microduplication 2q23.1. Eur. J. Hum. Genet. 2012, 20, 398-403.

39. Mullegama, S.V.; Rosenfeld, J.A.; Orellana, C.; van Bon, B.W.; Halbach, S.; Repnikova, E.A.; Brick, L.; Li, C.; Dupuis, L.; Rosello, M.; et al. Reciprocal deletion and duplication at 2q23.1 indicates a role for MBD5 in autism spectrum disorder. Eur. J. Hum. Genet. 2014, 22, 57-63.

40. Novara, F.; Rizzo, A.; Bedini, G.; Girgenti, V.; Esposito, S.; Pantaleoni, C.; Ciccone, R.; Sciacca, F.L.; Achille, V.; Della Mina, E.; et al. Mef2c deletions and mutations versus duplications: A clinical comparison. Eur. J. Med. Genet. 2013, 56, 260-265.

41. Willemsen, M.H.; Vulto-van Silfhout, A.T.; Nillesen, W.M.; Wissink-Lindhout, W.M.; van Bokhoven, H.; Philip, N.; Berry-Kravis, E.M.; Kini, U.; van Ravenswaaij-Arts, C.M.; Delle Chiaie, B.; et al. Update on Kleefstra syndrome. Mol. Syndromol. 2012, 2, 202-212.

42. Banka, S.; Lederer, D.; Benoit, V.; Jenkins, E.; Howard, E.; Bunstone, S.; Kerr, B.; McKee, S.; Lloyd, I.C.; Shears, D.; et al. Novel KDM6A (UTX) mutations and a clinical and molecular review of the X-linked Kabuki syndrome (KS2). Clin. Genet. 2015, 87, 252-258.

43. Bogershausen, N.; Wollnik, B. Unmasking Kabuki syndrome. Clin. Genet. 2013, 83, 201-211.

44. Elsea, S.H.; Girirajan, S. Smith-magenis syndrome. Eur. J. Hum. Genet. 2008, 16, 412-421.

45. Goh, K.I.; Cusick, M.E.; Valle, D.; Childs, B.; Vidal, M.; Barabasi, A.L. The human disease network. Proc. Natl. Acad. Sci. USA 2007, 104, 8685-8690.

46. Goh, K.I.; Choi, I.G. Exploring the human diseasome: The human disease network. Brief. Funct. Genomics 2012, 11, 533-542.

47. Jupiter, D.C.; VanBuren, V. A visual data mining tool that facilitates reconstruction of transcription regulatory networks. PLOS ONE 2008, 3, e1717.

48. Duhr, F.; Deleris, P.; Raynaud, F.; Seveno, M.; Morisset-Lopez, S.; Mannoury la Cour, C.; Millan, M.J.; Bockaert, J.; Marin, P.; Chaumont-Dubel, S. Cdk5 induces constitutive activation of 5-HT6 receptors to promote neurite growth. Nat. Chem. Biol. 2014, 10, 590-597.

49. Camarena, V.; Cao, L.; Abad, C.; Abrams, A.; Toledo, Y.; Araki, K.; Araki, M.; Walz, K.; Young, J.I. Disruption of mbd5 in mice causes neuronal functional deficits and neurobehavioral abnormalities consistent with 2q23.1 microdeletion syndrome. EMBO Mol. Med. 2014, 6, 1003-1015.

50. Tahir, R.; Kennedy, A.; Elsea, S.H.; Dickinson, A.J. Retinoic acid induced-1 (Rai1) regulates craniofacial and brain development in Xenopus. Mech. Dev. 2014, 133, 91-104.

51. Wei, H.; Alberts, I.; Li, X. The apoptotic perspective of autism. Int. J. Dev. Neurosci. 2014, 36, $13-18$.

(C) 2015 by the authors; licensee MDPI, Basel, Switzerland. This article is an open access article distributed under the terms and conditions of the Creative Commons Attribution license (http://creativecommons.org/licenses/by/4.0/). 